\title{
Tratamiento combinado de quimioterapia y cirugía en el tumor venéreo transmisible en caninos
}

\section{Combined treatment of chemotherapy and surgery in the transmissible venereal tumor in canines}

\author{
José Luis Sastre Torres ${ }^{1,3}$, Yunaika Mato Lorenzo ${ }^{1}$, Leonel Lazo Pérez ${ }^{1}$, \\ Carlos A. Bulnes Goicochea ${ }^{2}$
}

\section{Resumen}

Se realizó un estudio descriptivo con el objetivo de evaluar la efectividad de la combinación del tratamiento quirúrgico y quimioterapéutico en el tumor venéreo transmisible (TVT) en caninos. El estudio se realizó con casos llevados a consulta en la clínica veterinaria del municipio de Rodas, Provincia Cienfuegos, Cuba. La población estudiada fue de 57 perros diagnosticados con TVT. Un grupo $(n=14)$ recibió quimioterapia con sulfato de vincristina $(0.01 \mathrm{mg} / \mathrm{kg}$, vía endovenosa, tres veces con intervalos de 10 días $)$, un segundo grupo $(n=17)$ fue sometido a tratamiento quirúrgico y el resto $(n=26)$ recibió tratamiento quirúrgico inicial seguido de la quimioterapia, con la misma dosis y concentración que el primer grupo. Los resultados demuestran que la combinación de la cirugía y quimioterapia resultó en la total recuperación de los animales en el 95\% de los casos en un periodo de tres meses.

Palabras clave: quimioterapia; cirugía; perro; efectividad; neoplasia, TVT

\section{Abstract}

A descriptive study was conducted with the aim to evaluate the effectiveness of the combination of surgical and chemotherapeutic treatment in transmissible venereal tumor (TVT) in canines. The study was carried out with cases brought to consultation in the veterinary clinic of the municipality of Rodas, Cienfuegos Province, Cuba. The population studied was 57 dogs diagnosed with TVT. One group $(n=14)$ received chemotherapy with

\footnotetext{
${ }^{1}$ Facultad de Ciencias Agropecuarias. Universidad Central Martha Abreu de Las Villas, Cuba

${ }^{2}$ Universidad Técnica de Manabí, Ecuador

${ }^{3}$ E-mail: carmennatachaperez@gmail.com
}

Recibido: 4 de noviembre de 2018

Aceptado para publicación: 24 de mayo de 2019 
vincristine sulfate $(00.1 \mathrm{mg} / \mathrm{kg}$ intravenously, three times at 10 -day interval), a second group $(n=17)$ underwent surgical treatment and the rest $(n=26)$ received initial surgical treatment followed by chemotherapy, with the same dose and concentration as the first group. The results showed that the combination of surgery and chemotherapy obtained the total recovery of the animal in $95 \%$ of the cases in a period of three months.

Key words: chemotherapeutic; surgery; dog; effectiveness; neoplasia, TVT

\section{INTRODUCCIÓN}

El Tumor Venéreo Transmisible (TVT), también conocido como sarcoma infeccioso, granuloma venéreo, linfosarcoma transmisible o tumor de Sticker, es un tumor retículo endotelial benigno del perro que afecta principalmente a los genitales externos y ocasionalmente a los genitales internos. Aunque el TVT posee una distribución mundial, es una neoplasia de alta frecuencia en zonas tropicales y subtropicales, especialmente en áreas urbanas con grandes poblaciones de perros callejeros (De la Sota et al., 2017).

La localización más frecuente del TVT en machos es desde la parte caudal del pene hasta el bulbo del glande y en hembras en la conjunción de la vagina y el vestíbulo. Los signos asociados con el tumor pueden ser confundidos con afecciones urinarias y con prostatitis (Peña et al., 2017). Entre las formas de transmisión se encuentran el olfateo y el lamido que permiten la implantación de las células tumorales en zonas extragenitales (Ojeda et al., 2016). Los tumores primarios usualmente se localizan en la mucosa de los genitales externos, aunque se han reportado casos de implantación extragenital primaria sin compromiso genital previo (Crossley y Ramírez, 2017). Las metástasis localizadas en pulmones, vísceras abdominales y en el sistema nervioso central son raras (Sánchez et al., 2017).

En el tratamiento del tumor se utiliza la cirugía, bioterapia, inmunoterapia y la quimioterapia. La cirugía se considera un procedi- miento efectivo, aunque se pueden presentar recidivas (Benavides et al., 2017). La quimioterapia con sulfato de vincristina reporta la remisión del tumor hasta en el $90 \%$ de los perros tratados, debido a que induce a una apoptosis a las células tumorales (Marín et al., 2017); sin embargo, este medicamento produce efectos colaterales que afectan el bienestar del animal (Carvajal et al., 2016). Por otro lado, la inmunoterapia disminuye los efectos secundarios de tratamientos agresivos como el de la vincristina (Espinosa et al., 2014).

El objetivo de este trabajo fue evaluar la efectividad de la combinación del tratamiento quirúrgico y quimioterapéutico en el tumor venéreo trasmisible en caninos.

\section{Materiales y Métodos}

El estudio se realizó en la clínica veterinaria del municipio de Rodas, región central de Cuba, con 57 caninos diagnosticados clínicamente con TVT (33 machos y 24 hembras). La muestra en estudio correspondió a los animales remitidos a consulta en un lapso de tres meses. Los animales tenían entre 2 y 10 años y fueron distribuidos en tres grupos etarios: 2-4, 5-7 y 8-10 años. El 51\% de estos perros fue mestizo y el resto fue considerado de raza, habiendo más de 10 razas, sin que hubiera una mayor frecuencia en alguna de ellas (Cuadro 1).

Para el estudio, 14 perros recibieron quimioterapia como tratamiento, utilizando sulfato de vincristina en dosis de $0.01 \mathrm{mg} / \mathrm{kg}$ 
Cuadro 1. Distribución de perros con tumor venéreo trasmisible según grupo etario. Rodas, Cuba, 2017

\begin{tabular}{ccccc}
\hline $\begin{array}{c}\text { Edad } \\
\text { (años) }\end{array}$ & $\mathrm{n}$ & Proporción & Error & Varianza \\
\hline $2-4$ & 26 & $0.45^{\mathrm{a}}$ & 0.097 & 0.248 \\
$5-7$ & 22 & $0.38^{\mathrm{a}}$ & 0.104 & 0.237 \\
$8-10$ & 8 & $0.14^{\mathrm{b}}$ & 0.123 & 0.121 \\
$>10$ & 1 & $0.02^{\mathrm{c}}$ & 0.131 & 0.017 \\
\hline
\end{tabular}

Proporciones con letras desiguales dentro de columnas son estadísticamente diferentes $(p<0.001)$

de peso vivo (PV), diluido en $100 \mathrm{ml}$ de solución salina al $0.9 \%$, administrada por vía endovenosa, en tres oportunidades a intervalos de 10 días. Carvajal et al. (2017) sostienen que la administración de dosis mínima de vincristina disminuye los efectos tóxicos de la droga en los animales tratados. Un segundo grupo de 17 perros fue sometido a tratamiento quirúrgico y los 26 perros restantes recibieron tratamiento quirúrgico inicial seguido de la con la misma dosis y concentración que el primer grupo.

El grado de las lesiones era similar en los animales del estudio. El tratamiento quirúrgico consistió en la remoción de la masa tumoral y de tejidos subyacentes, se cauterizó la zona donde se encontraban los tumores y se castró a los machos y se practicó la ovariohisterectomía a las hembras. A todos los animales se les hizo el seguimiento clínico durante tres meses posteriores al tratamiento. Se consideró como criterio satisfactorio la recuperación total del animal y no satisfactorio la presentación de recidivas.

La información obtenida se registró en una base de datos creada al efecto de esta investigación. Para el análisis de los datos se utilizó la comparación múltiple de proporciones que estima la diferencia a partir de la construcción de intervalos de confianza. Para ello se empleó el programa COMPAPROP v.2.0.1 (Castillo y Miranda, 2014).
El estudio cumple con las recomendaciones y normativas nacionales e internacionales sobre el bienestar de los animales utilizados con propósitos científicos, contenidas en los reglamentos establecidos por la Red Cubana de Comités Institucionales para el Cuidado y Uso de Animales (Red CICUAL, s.f.)

\section{Resultados y Discusión}

La frecuencia de animales afectados con TVT según el rango de edad se muestra en el Cuadro 1. La mayor frecuencia de casos se presentó en perros entre 2 y 7 años $(p<0.001)$.

Según De la Cruz et al. (2015), el TVT se presenta mayormente desde que el animal inicia la pubertad y tiene contacto con hembras en estro o con machos dominantes, quienes por su comportamiento tratan de montar a otros machos. Hernández (2015) demuestra que el TVT se produce en edades tan tempranas como las encontradas en esta investigación, aunque refiere que el tiempo de riesgo se incrementa con la edad del animal; así mismo, Huamán (2016) encontró una mayor frecuencia de presentación de tumores de este tipo en animales entre los 9 y los 12 años.

El sexo, no influyó significativamente en la frecuencia de aparición de estos tumores, lo que coincide con los resultados obtenidos 
Cuadro 2. Efectividad del tratamiento quirúrgico y quimioterapéutico en el tratamiento del tumor venéreo transmisible en caninos del municipio de Rodas, Cuba

\begin{tabular}{lccccc}
\hline Tratamientos & $\begin{array}{c}\text { Animales } \\
\text { tratados }\end{array}$ & $\begin{array}{c}\text { Animales } \\
\text { recuperados }\end{array}$ & Proporción & Error & Varianza \\
\hline Quirúrgico + & 26 & 24 & $0.92^{\mathrm{a}}$ & 0.05 & 0.07 \\
quimioterapia & & 8 & $0.57^{\mathrm{b}}$ & 0.17 & 0.24 \\
Quimioterapia & 14 & 2 & $0.12^{\mathrm{c}}$ & 0.23 & 0.10 \\
Quirúrgico & 17 & 24 &
\end{tabular}

Proporciones con letras desiguales dentro de columnas son estadísticamente diferentes $(p<0.001)$

por Medina et al. (2017) y Murcia et al. (2017); sin embargo, se encontró diferencia significativa en el aspecto racial, donde hubo una mayor frecuencia en animales mestizos $(49.2 \%)$, con relación al resto de las razas $(\mathrm{p}<0.05)$. De la Cruz et al. $(2015)$ no encontraron una predisposición racial; sin embargo, se reporta que la presencia de TVT está influenciada por el descuido o ignorancia en la crianza de mascotas, así como por una elevada presencia de perros callejeros, ya sea, en estado de abandono o por el poco control por parte de sus dueños, quienes permiten que el animal permanezca gran parte del día en la vía pública (Benavides et al., 2017; Torres et al., 2017). Los animales del presente estudio provenían de zonas de la ciudad donde se evidencia una alta concentración de perros callejeros, o que en su mayoría tienen un acceso libre a la calle.

La proporción de razas puras de Cuba es mínima en comparación con países desarrollados (Torres y Fabré, 2017). Este hecho pudiera justificar la baja proporción de animales de raza con diagnóstico de TVT en el presente estudio, en contradicción con los hallazgos de Ojeda et al. (2016), quienes afirman que, tanto en perros como en gatos de raza, se registra el doble de incidencia de tumores malignos, en comparación con animales mestizos.
La ubicación de las lesiones en todos los casos estudiados fue genital y se manifestó desde el punto de vista morfológico por la presencia de masas tumorales de aspecto multilobulado con forma de coliflor, friables y de color rosado con tonalidades dependientes del daño circulatorio. En algunas hembras se observaron descargas vulvares de tipo sanguinolento (Figura 1A). En los machos, las lesiones se ubicaron en la mayoría de los casos a nivel de la base del pene, como también a nivel de glande y prepucio (Figura 1B).

El Cuadro 2 muestra la efectividad de los tratamientos evaluados. El tratamiento combinado de cirugía seguido de quimioterapia resultó ser el más efectivo $(\mathrm{p}<0.001)$. Estos resultados pueden estar relacionados con el estado inmunitario de los pacientes o con alguna expresión genética propia del tumor (Medina et al., 2017; Vinueza et al., 2017).

El uso del sulfato de vincristina para el tratamiento del TVT es aún controversial, a pesar de que se conoce que consiste en un alcaloide que interfiere con la formación de los microtúbulos, deteniendo la metafase y los procesos de proliferación celular (Sanabria et al., 2017), además de favorecer una mayor infiltración de linfocitos $\mathrm{CD}^{+}$(Avilés et al., 2017). El sulfato de vincristina se utiliza 

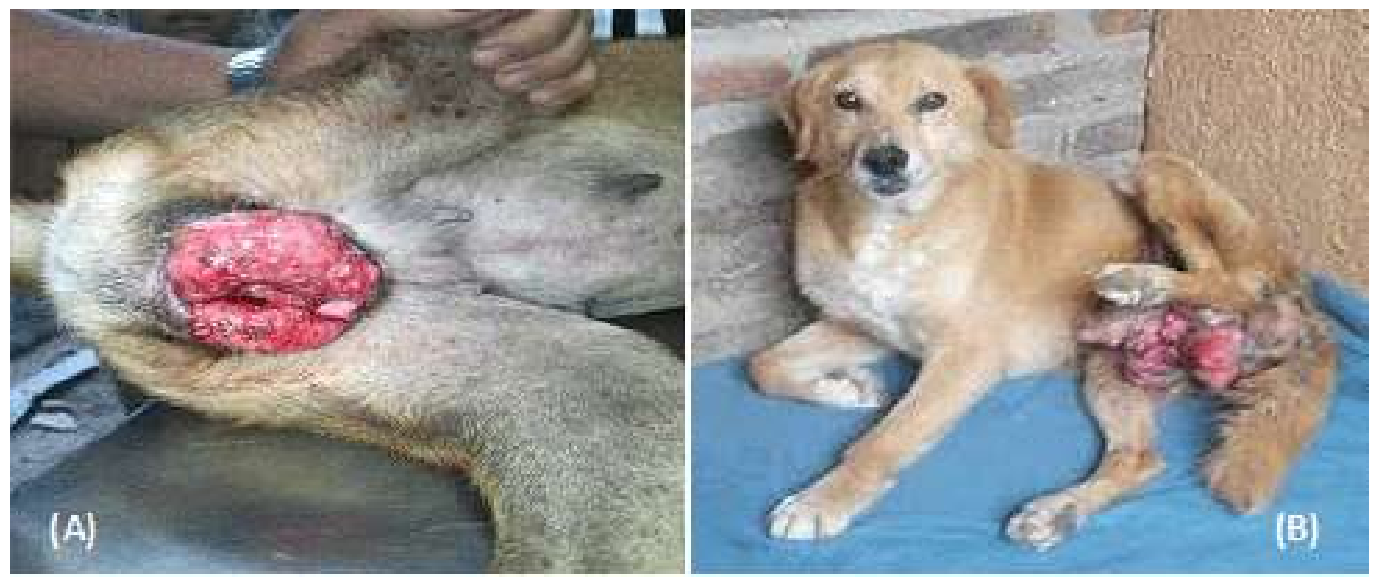

Figura 1.- Casos clínicos de tumor venéreo transmisible (TVT). A: Lesión vulvar en una hembra mestiza de 3 años. B: Lesión de aspecto en forma de coliflor en la base del pene en un perro macho mestizo de 5 años

con otros agentes antineoplásicos, especialmente en procesos linfoproliferativos con el fin de tratar la trombocitopenia (Vistín, 2017). Ramírez et al. (2016) emplearon vincristina como agente único a dosis semanal de 0.025 $\mathrm{mg} / \mathrm{kg}$, obteniendo buenos resultados y con efectos colaterales mínimos, coincidiendo con los resultados del presente estudio. Por otro lado, Quiroga y Espinosa (2016) indican que debe iniciarse tan pronto haya ocurrido el procedo de cicatrización de la cirugía.

El presente estudio ofrece resultados favorables en la aplicación de tratamiento quirúrgico combinado con quimioterapia, coincidiendo con el estudio de Núñez et al. (2017).

\section{Literatura Citada}

1. Avilés CG, Palomino SR, Claros GT, Gasca AG 2017. Presentación de TVT en un canino de raza Pitbull con paraplejia (alternativas en el tratamiento de la paraplejia): reporte de caso. REDVET 18(11). [Internet]. Disponible en: http://www.veterinaria.org/revistas/ redvet/n111117/111716.pdf

2. Benavides AA, Murcia EH, Quevedo MA, Suaza DM. 2017. Autohemo-terapia como adyuvante en el tratamiento del tumor venéreo transmisible (TVT) en canino: descripción de un caso clínico. REDVET 18(5). [Internet]. Disponible en: http://www.veterinaria.org/revistas/ redvet/n050517/051702.pdf

3. Carvajal Santana DA, García Cuellar AR, Maltes Sánchez JM, Ortiz Fajardo DA, Valencia Hernández AF. 2016. Efecto del sulfato de vincristina sobre las células hematológicas en un paciente con tumor venéreo transmisible (TVT). REDVET 17(4). [Internet]. Disponible en:

4. Castillo Y, Miranda I. 2014. COMPAPROP: sistema para comparación de proporciones múltiples. Rev Protección Veg 29: 231-234.

5. Chang G. 2016. Frecuencia de neoplasias en caninos de 0 a 5 años de edad diagnosticados histopatológicamente en el Laboratorio de Histología, Embriología y Patología Veterinaria de la Facultad de Medicina Veterinaria de 
la Universidad Nacional Mayor de San Marcos. Periodo 2003-2014. Tesis de Médico Veterinario. Lima, Perú: Univ. Nacional Mayor de San Marcos. 63 p.

6. Crossley R, Ramírez JA. 2017. Tumor venéreo transmisible canino de presentación atípica. Reporte de caso clínico. Rev Med Vet Zoot 64: 78-90.

7. De la Cruz S, Quijano-Hernández IA, Del Ángel J, Martínez JS, VictoriaMora JM, Barbosa-Mireles MA. 2015. Respuesta del tumor venéreo transmisible canino a presentaciones de vincristina de patente y genérica. Rev Inv Vet Perú 26: 587-595. doi: 10.15381/rivep.v$26 \mathrm{i} 4.11212$

8. De la Sota P, Dàmico G, Adagio LM, Noia MA, Gobello MC. 2017. Tumor venéreo transmisible en el perro. Cienc Vet 6: 42-29.

9. Espinosa A, Muñoz L, Nastar R. 2014. Inmunoterapia como tratamiento alternativo para tumor venéreo transmisible: reporte de caso clínico. FAGROPEC 6: 46-55.

10. Hernández AJ. 2015. Efecto de la terapia autóloga con linfocitos T CD8+ estimulados con antígeno tumoral total $\mathrm{E}$ IL-21 en caninos con tumor venéreo transmisible. Tesis de Maestría. México: Univ. Autónoma de Nuevo León. 93 p.

11. Marín E, Quiacha B, Zambrano PS, Hernández C, Zea P. 2017. Reporte de caso: tumor venéreo transmisible y su respuesta quimioterapéutica apoyada con homeoterapia en canino. REDVET 18(7). [Internet]. Disponible en: http:// www.veterinaria.org/revistas/redvet/ n070717/071710.pdf

12. Medina I, Puicón V, Sandoval N. 2017. Frecuencia de tumores en piel de caninos diagnosticados histopatológicamente en el Laboratorio de Patología Veterinaria de la Universidad Nacional Mayor de San Marcos. Rev Inv Vet Perú 28: 448-454. doi: 10.15381/ rivep.v28i2.13065
13. Murcia EH, Artunduaga CA, Hurtado LE, Osorio AF, Pedroza LA. 2017. Tumor venéreo transmisible (TVT) de localización extragenital en canino (linfonodos regionales). Descripción de un caso clínico. REDVET 19(5). [Internet]. Disponible en: http:// www.veterinaria.org/revistas/redvet/ n050518/051827.pdf

14. Ojeda J, Alfaro A, Moroni M, Camacho V, Martínez J, Noro M. 2016. Disseminated transmissible venereal tumor on skin, eyelids and genital in a male dog: case report. Arch Med Vet 48: 119-123.

15. Ortiz WF, Duque L, Sandoval CA, Castillo CE, Bejarano OE. 2017. Efecto de vincristina como método eficaz en tumor venéreo transmisible. REDVET 18(10). [Internet]. Disponible en: http://www.veterinaria.org/revistas/ redvet/n101017/101720.pdf

16. Peña G, Vidal F, del Toro R, Hernández R. 2017. Empleo del sulfato de vincristina, vía subcutánea, para el control del tumor venéreo transmisible canino: estudio retrospectivo de siete casos en Camagüey, Cuba. Rev Inv Vet Perú 28: 750-756. doi: 10.15381/ rivep.v28i3.13363

17. Quiroga I, Espinosa A. 2016. Tratamientos alternativos en tumor venéreo transmisible en caninos. Tesis de Grado. Bogotá, Colombia: Univ. De la Salle. 52 p.

18. Ramírez FT, Sotto LG, Manjarres NR, Artunduaga L. 2015. Reporte de caso: tumor venéreo transmisible en perro. REDVET 16(1). [Internet]. Disponible en: http://www.veterinaria.org/revistas/ redvet/n010115/011506.pdf

19. Red CICUAL. s.f. [Internet]. CICUAL IPK. Cuba. Disponible en: https:// instituciones.sld.cu/ipk/red-cubana-decomites-institucionales-de-cuidado-yuso-de-animales-de-laboratorio/

20. Sanabria JJ, Lozada ML, Salinas MA, Camacho FA, Bautista LK, Ángel JV. 2017. Tumor venéreo transmisible dise- 
minado en canino. Reporte de Caso. REDVET 18(12). [Internet]. Disponible en: http://www.veterinaria.org/revistas/ redvet/n121217/121751.pdf

21. Sánchez I, Canizales S, Casanova C, Guzmán A, Ramírez E. 2017. Aparición primaria de tumor venéreo transmisible (TVT) en cavidad nasal de un canino hembra. Reporte de caso clínico. REDVET 18(3). [Internet]. Disponible en: http://www.veterinaria.org/revistas/ redvet/n030317/031708.pdf

22. Torres M, Fabré Y, Rodríguez, J, Calaña L. 2017. Frecuencia de presentación de tumores cutáneos en caninos de la provincia La Habana entre 2009-2013. REDVET18(3). [Internet]. Disponible en: http://www.veterinaria.org/revistas/ redvet/n030317/031701.pdf

23. Vistín G. 2017. Prevalencia de Tumor Venéreo Transmisible (TVT) en perros sexualmente activos en la ciudad de Guaranda. Tesis de Médico Veterinario Zootecnista. Guarandá, Ecuador: Univ. Estatal de Bolívar. $74 \mathrm{p}$.

24. Vinueza RL, Cabrera F, Donoso L, Pérez J, Díaz R. 2017. Frecuencia de neoplasias en caninos en Quito, Ecuador. Rev Inv Vet Perú 28: 92-100. doi: 10.15381/rivep.v28i1.12931 